\title{
FLORA DA RESERVA BIOLÓGICA GUARIBAS, PARAÍBA: FAMÍLIA HUMIRIACEAE JUSS
}

\author{
FLORA OF THE GUARIBAS BIOLOGICAL RESERVE, PARAÍBA: FAMILY HUMIRIACEAE JUSS
}

\begin{abstract}
Monise Gomes Nunes ${ }^{1}$ Rita Baltazar de Lima $\dagger^{2}$

\section{Resumo}

A família Humiriaceae possui oito gêneros e cerca de 65 espécies, com distribuição tropical, contando com um representante no continente africano. As Humiriaceae são plantas lenhosas, arbóreas ou arbustivas, apresentando flores com estames distribuídos em até dois verticilos e ovário súpero. $\mathrm{O}$ trabalho consiste na descrição e ilustração do material examinado, pertencente à coleção do Herbário JPB. A Reserva Biológica Guaribas, inserida no bioma Mata Atlântica, está localizada nos municípios de Rio Tinto e Mamanguape, no Litoral Norte da Paraíba, para qual foi registrada apenas a ocorrência da espécie Sacoglottis mattogrossensis Malme.
\end{abstract}

Palavras-chave: Humiriaceae, Floresta Atlântica, REBIO Guaribas, Sacoglottis mattogrossensis.

\begin{abstract}
The family Humiriaceae has eight genera and about 65 species, with tropical distribuition, with one representative on the African continent. The Humiriaceae are woody plants, trees or shrubs, showing flowers with stamens distributed in up to two whorls and superior ovary. The work consists of the description and illustration of the examined material, belonging to the collection of the Herbarium JPB. The Biological Reserve Guaribas, inserted in the Atlantic Forest, is located in the municipalities of Rio Tinto and Mamanguape, on the north coast of Paraiba, for which was only registered the occurrence of the species Sacoglottis mattogrossensis Malme.
\end{abstract}

Keywords: Humiriaceae, Atlantic Forest, REBIO Guaribas, Sacoglottis mattogrossensis.

1 Graduanda em Ciências Biológicas/UFPB. E-mail: monise.gnunes@gmail.com

2 Departamento de Sistemática e Ecologia, CCEN, Universidade Federal da Paraíba, C. Postal 5065, Cidade Universitária, CEP 58051-970, João Pessoa, PB, Brasil.

† Falecida. 


\section{Tratamento taxonômico da família na REBIO Guaribas}

Humiriaceae Juss. Fl. Bras. Merid. 2(13): 87. 1829.

Plantas lenhosas, arbóreas ou arbustivas. Folhas simples, pecioladas, alternas, elípticas, coriáceas, glabras. Inflorescência axilar paniculada. Flores 5-meras, diclamídeas, actinomorfas, andróginas; cálice gamossépalo; corola dialipétala; androceu gamostêmone com anteras rimosas; ovário súpero. Fruto drupa globosa.

Sacoglottis mattogrossensis Malme. Ark. Bot. 22A(7): 91928.

(Fig. 1)

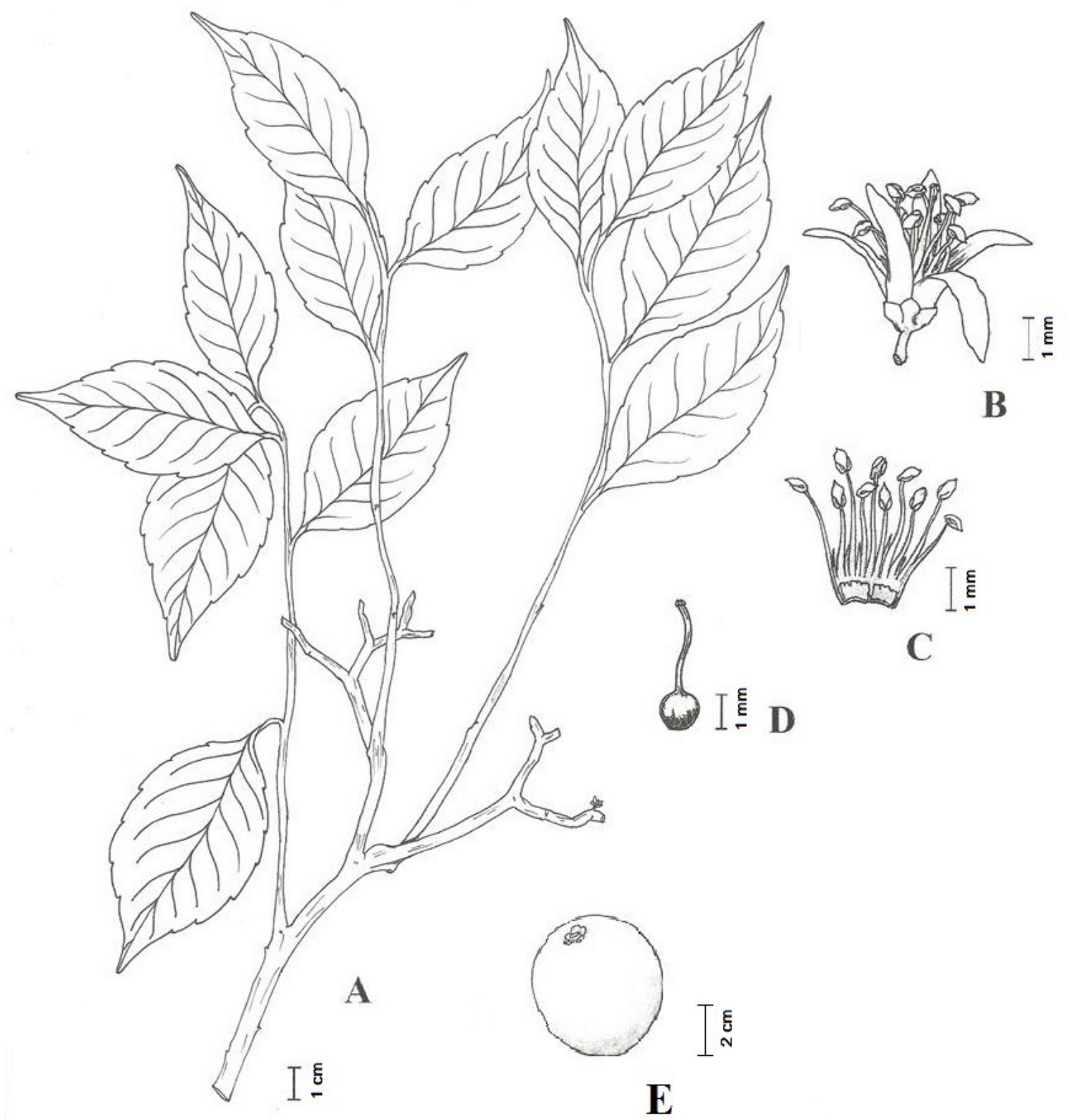

Figura 1. Sacoglottis mattogrossensis Malme. A. Hábito; B. Flor; C. Androceu; D. Gineceu (O.T. Moura 687). 
Árvores ou arbustos. Folhas elípticas, base atenuada, margem crenada, ápice acuminado a cuspidado, coriáceas, glabras, 3,4-8,7 cm compr., 2,3-4,6 cm larg. Inflorescência axilar paniculada. Flores 5-meras, 3-6 mm compr.; cálice com sépalas esverdeadas, ápice agudo, $1-5 \mathrm{~mm}$ compr.; corola com pétalas amarelas, lanceoladas, 3-5 mm compr.; 10-14 estames, heterodínamos, filetes unidos na base em até dois verticilos, anteras rimosas; 5-carpelar, placentação axilar. Fruto drupa globosa.

Material Examinado: Brasil, Paraíba: Mamanguape: Reserva Guaribas, 27/ IV/1993, fr., Barbosa et al 1346 (JPB).

Material Adicional: Brasil, Paraíba: Bayeux: Mata do Xém-Xém. XII/2ooo, fl. e fr., G. Freitas et al 53 (JPB); Cabedelo: Mata do Amém, 15/X/1999, fr., A.F. Pontes 228 (JPB). Restinga de Camboinha, o2/II/1969, fl. e fr., L. Xavier s/n (JPB 2111). Conde: Praia de Jacumã, 18/XI/1991, fl., O.T. Moura 687 (JPB); Tabatinga, 20/IX/1994, fl., O.T. Moura 1408 (JPB). João Pessoa: 17/VII/1994, fr., O.T. Moura s/n (JPB 24088); Antiga Fazenda de Mangabeira. 22/XI/1994, fl. e fr., O.T. Moura 1456 (JPB); 25/IX/1994, fl., O.T. Moura s/n (JPB 22701); 17/X/1994, fl., O.T. Moura 1432 (JPB); 17/X/1994, fl., O.T. Moura 1433 (JPB); Mangabeira VII, 4/IX/1994, fl., O.T. Moura 1088 (JPB); UFPB, Campus I, 27/ III/1987, fl., C.A.B. Miranda 308 (JPB); 10/V/1993, P.C.G. Neto 23 (JPB); 26/IX/1994, fl., O.T. Moura s/n (JPB 22704); Praia da Penha, estrada que liga à Vila dos Pescadores, 29/X/1994, fr., O.T. Moura 1448 (JPB); Sem local especificado, 1994, fl., O.T. Moura s/n (JPB 22702). Santa Rita: Mata, o6/VII/1992, fl., M.F. Agra \& G. Góis 1547 (JPB); Sem local especificado, 22/IX/1987, fl., M.F. Agra 629 (JPB).

Distribuição geográfica: no Brasil ocorre nos estados do Amazonas, Bahia, Distrito Federal, Maranhão, Mato Grosso, Mato Grosso do Sul, Minas Gerais, Pará, Paraíba, Pernambuco, Rio de Janeiro, Rondônia, Santa Catarina, São Paulo (GIORDANO, 1996; MEDEIROS, 2014; NETO, 2008; REITZ, 1967).

Fenologia: encontrada florida e frutificado em todo o ano.

Nomes vernáculos: pitomba de morcego, pitomba brava, oiticica (Paraíba).

O gênero Sacoglottis Mart. abrange 11 espécies, descrito pela primeira vez por Martius em 1827 e está distribuído no Brasil, Colômbia, Costa Rica, Honduras e Panamá (TROPICOS 2014). No Brasil é possível encontrar cinco espécies, sendo uma delas endêmica, e está distribuído em quase todo o território nacional (MEDEIROS et al., 2014; NETO e BOVE, 2008).

Na Paraíba, duas espécies podem ser encontradas, Sacoglottis guianensis Benth. e Sacoglottis mattogrossensis Malme, esta última com registro na REBIO Guaribas (BARBOSA et al., 2011). A espécie $S$. mattogrossensis foi descrita pela primeira vez por Malme em 1928 e está distribuída na América do Sul, no Brasil, Bolívia, Guiana 
e Venezuela (TROPICOS, 2014). No Brasil, a espécie S. mattogrossensis ocorre na Amazônia, Cerrado e Mata Atlântica, em florestas de terra firme, matas fechadas ou em campos e restingas, e está distribuída em quase todo o território nacional (GIORDANO, 1996; MEDEIROS, 2014). A espécie é caracterizada pelas folhas cartáceas ou coriáceas flexíveis, estruturas vegetativas e reprodutivas glabras, com frutos globosos (GIORDANO, 1996; NETO e LIMA, 2008).

\section{AGRADECIMENTOS}

À curadora e professores do Herbário Lauro Pires Xavier (JPB) pela disponibilização deste para a análise das coleções. Aos colegas do TAXON. À Professora Rita Baltazar de Lima pelo tempo, incentivo e colaboração para que esse trabalho pudesse ser realizado.

\section{REFERENCIAS}

AUBLET, J. B. C. F. 1775 - Houmiri balsamifera et Vantanea guianensis. In: Histoire des plantes de la Guiane Frainçoise. Paris, 1: 564-572, t. 225-229.

BARBOSA, M. R. V.; THOMAS, W. W.; ZÁRATE, E. L. P.; LIMA, R. B.; AGRA, M. F.; LIMA, I. B.; PESSOA, M. C. R.; LOURENÇO, A. R. L.; DELGADO-JR., G. C.; PONTES, R. A. S.; CHAGAS, E. C. O.; VIANA, J. L.; GADELHA-NETO, P. C.; ARAÚJO, A. A. M.; FREITA, G. B.; LIMA, J. R.; SILVA, F. O.; VIEIRA, L. A. F.; PEREIRA, L. A.; COSTA, R. M. T.; DURÉ, R. C. e SÁ, M. G. V. 2011 - Checklist of the vascular plants of the Guaribas Biological Reserve, Paraíba, Brazil. Revista Nordestina de Biologia, 20(2): 79-106.

BOVE, C. P. 1997 - Phylogenetic analysis of Humiriaceae with notes on the monophyly of Ixonanthaceae. Journal Complishment Biology, 2: 19-24.

CUATRECASAS, J. 1961 - A Taxonomic revision of the Humiriaceae. Contr. U.S. Nat. Herb, 35(2): 25-214.

CUATRECASAS, J. 1990 - Miscellaneous notes on Neotropical Flora XVIII. New species in the Humiriaceae. Phytologia, 68(4): 260-264.

CUATRECASAS, J. 1993 - Miscellaneous notes on Neotropical Flora XXI. A new species of Humiriastrum from Brazil. Phytologia, 75(3): 235-238. 
FANTINATI, M. R.; PONTES, A. F.; MENDONÇA, F. B. e COSTA, A. D. C. 2004 Flora da Serra do Cipó, Minas Gerais: Humiriaceae. Boletim de Botânica, 22(1): 29-33.

ORDANO, L. C. S. 1996 - Estudo taxonômico de Humiriaceae no estado do Rio de Janeiro, Brasil. Arch. Jard. Bot. Rio de Janeiro, 34(2): 5-50.

GIORDANO, L. C. S. e BOVE, C. P. 2008 - Taxonomic considerations and amended description of Humiriastrum spiritu-sancti, Humiriaceae. Rodriguésia, 59(1): 151-154.

HERRERA, F.; MANCHESTER, S. R.; JARAMILLO, C.; MACFADDEN, B. e SILVACAMINHA, S. A. 2010 - Phytogeographic history and phylogeny of the Humiriaceae. International Journal of Plant Sciences, 171(4):392-408.

HERRERA, F.; MANCHESTER, S. R.; VÉLEZ-JUARBE, J. e JARAMILLO, C. 2014 Phytogeographic History of the Humiriaceae (Part 2). Int. J. Plant Sci, 175(7).

JUSSIEU, A. D. 1829 - Humiriaceae. In:St. HILLAIRE, A. Flora Brasiliae Meridionalis. Paris, 2: 87 .

MEDEIROS, H.; AMORIM, A. M. A. e HOLANDA, A. S. S. Humiriaceae in Lista de Espécies da Flora do Brasil. Jardim Botânico do Rio de Janeiro. Disponível em: <http:// floradobrasil.jbrj.gov.br/jabot/floradobrasil/FB129>. Acesso em: 20 Jan. 2014.

NETO, A. H. S. e LIMA, R. B. 2008 - Flora paraibana: família Humiriaceae Juss. Revista Nordestina de Biologia, 17(1): 3-10.

PEREIRA, C. 1966 - Contribuição ao conhecimento da flora do estado da Guanabara. Família Humiriaceae. Rodriguésia, 25(37): 13-22.

PIRANI, J. R.; MELLO-SILVA, R. D. e GIULIETTI, A. M. 2003 - Flora de Grão-Mogol, Minas Gerais, Brasil. Boletim de Botânica da Universidade de São Paulo, 21(1): 1-24.

REITZ, P. R. 1967 - Humiriáceas. In: Flora Ilustrada Catarinense. Parte I: As Plantas. Itajaí: Herbário Barbosa Rodrigues, 10.

TROPICOS.ORG. Missouri Botanical Garden. Disponível em: <http://www.tropicos. org/Name/40028382>. Acesso em: 16 Out 2014.

URBAN, I. 1877 - Humiriaceae. In: MARTIUS, K. P.; EICHLER, A. G e URBAN, I. Flora brasiliensis. 12(2): 434-454, tab. 92-96. 